\title{
Analysis of D2D Communications over Gamma/Nakagami Fading Channels
}

\author{
Zakir Hussain \\ National University of \\ Computer and Emerging \\ Sciences \\ Karachi, Pakistan
}

\author{
Asim ur Rehman Khan \\ National University of \\ Computer and Emerging \\ Sciences \\ Karachi, Pakistan
}

\author{
Haider Mehdi \\ National University of \\ Computer and Emerging \\ Sciences \\ Karachi, Pakistan
}

\author{
Syed Muhammad Atif Saleem \\ National University of Computer \\ and Emerging Sciences \\ Karachi, Pakistan
}

\begin{abstract}
In this paper, we investigate the outage probability, channel capacity and symbol error rate (SER) performance of device-to-device (D2D) communication systems. The D2D communication system is affected by several co-channel interferers. Gamma fading channel is considered for the D2D communication system. The channel for the co-channel interference is assumed to be Nakagami faded. An expression for the probability density function (PDF) of the signal-tointerference ratio (SIR) is presented. The PDF is a function of distances between various devices in the D2D system, path-loss, channel fading conditions and signal powers. Based on the PDF expression, we present the expressions for the outage, channel capacity and SER. With the help of numerical results the performance of D2D communication system is discussed under various conditions of interference, path-loss and channel fading.
\end{abstract}

Keywords-channel capacity; co-channel interference; D2D communication; outage; gamma fading; Nakagami fading

\section{INTRODUCTION}

The rapid growth of interconnected wireless devices needs efficient wireless networks to fulfill demands of high data rates [1-5]. For this purpose device-to-device (D2D) communication system is one of the promising technologies introduced [6-10]. D2D communication is a $5 \mathrm{G}$ cellular communication standard that facilitates nearby active devices to communicate directly without going through a base-station (BS) [11-15]. Services such as multimedia sharing, online gaming and cloud computing etc., have become vital part of daily communications. D2D communication is one of the communication systems which enable these services. D2D communication can also be used to encounter the excessive user density [16-20]. Due to the large number of wireless devices always competing for the limited wireless resources, any lack of coordination or management between them, may result in a scenario of unintentionally jamming of wireless signals due to co-channel interference (CCI). CCI causes degradation in the performance of the system. Therefore, effects of CCI should be taken into consideration while analyzing the performance of wireless communication systems [21-23]. D2D communication system also suffers from CCI [24]. Communication system performance can be analyzed using well known metrics like outage performance, channel capacity and symbol error rate (SER) [25, 28]. Outage performance of multi-antenna D2D system is studied in [25] over a Rayleigh fading channel with CCI. In [26], authors investigate outage probability of D2D multichannel systems over Raleigh fading channels in the presence of interference. The outage analysis of D2D systems with Suzuki fading channel and interference is presented in [27]. Channel capacity of D2D communication system is studied in [28] over a Rician fading channel and CCI. In [29], channel capacity of D2D system is presented over a Rician/Rayleigh channel and CCI. In [29], SER analysis of M-ary phase-shift keying (MPSK) for D2D systems over a Rician/Rayleigh channel is studied.

In this paper, we study outage probability, channel capacity and SER performance of D2D communication systems with multiple co-channel interferers. Based on the probability density function (PDF) expression of signal-to-interference (SIR) of the D2D system, expressions for the outage, channel capacity and SER are presented. Channel for D2D communication system is assumed to be Gamma distributed. The generality of Gamma distribution makes it feasible for the analysis of D2D communication system. It can be used to model severe channel fading conditions. In this work, we assume channel for the interferers to be Nakagami faded.

\section{SYSTEM MODEL}

We consider an interference limited system where an active pair of D2D communication devices is communicating [30]. System layout is shown in Figure 1. We consider several cochannel interferers in the system. These interferers are assumed to be equidistant from the D2D receiver. The channel for cochannel interferers is considered to be Nakagami faded. Channel gains for the co-channel interferers are assumed to be independent and identically distributed (IID) [31].

The PDF of Nakagami fading is given as [32]

$$
f_{X}(x)=\frac{2}{\Gamma(m)}\left(\frac{m}{\Omega}\right)^{m} x^{2 m-1} \exp \left(\frac{-m}{\Omega} x^{2}\right), \quad m \geq \frac{1}{2}, \Omega>0, x>0
$$

where $m$ is the fading parameter which describes the severity of the channel fading conditions, $\Omega$ is the average power of fading and $\Gamma($.$) is gamma function [32,33]$. The channel for 
the D2D pair is assumed to be gamma distributed. The PDF of gamma distribution is [34]:

$$
f(x)=\frac{e^{-\frac{x}{\theta}} x^{k-1}}{\theta^{k} \Gamma(k)}, \quad x \geq 0, \theta>0, k>0,
$$

where $k$ is the shape parameter, related to the intensity of the channel fading and $\theta$ is the scale parameter of gamma distribution [35]. Path-loss is another factor which affects the performance of communication systems. In this paper, we consider a simplified path-loss model. The received power of the D2D communication system is:

$$
S_{d}=P_{1}\left(\frac{\lambda}{4 \pi c_{0}}\right)^{2}\left(\frac{c_{0}}{c}\right)^{a}
$$

where $P_{1}$ is the D2D signal power, $c$ is the distance between sender and receiver of $\mathrm{D} 2 \mathrm{D}$ pair, $\lambda$ is the wavelength, $a$ is the path-loss exponent $(2 \leq a \leq 5)$ and $c_{0}$ is the reference distance (1 to 100 meters) [36]. Similarly, power of the $i$-th interferer at the $\mathrm{D} 2 \mathrm{D}$ receiver is given as

$$
I=P_{2}\left(\frac{\lambda}{4 \pi d_{0}}\right)^{2}\left(\frac{d_{0}}{d}\right)^{b}
$$

In (2), $P_{2}$ is the interference signal power, $d$ is the distance between the receiver of D2D pair and the $i$-th interferer, $b$ is the path-loss exponent and $d_{0}$ is the reference distance. Using expressions (1) and (2) the SIR of considered D2D communication system is given as

$$
\gamma=\frac{h^{2}}{g \sum_{i=1}^{N} \alpha_{i}^{2}}, \quad g=\frac{P_{2}}{P_{1}}\left(\frac{c^{a}}{d^{b}}\right) \frac{\left(c_{0}\right)^{2-a}}{\left(d_{0}\right)^{2-b}},
$$

where $h$ is the independent gamma fading variable of the desired D2D signal, $\alpha_{i}$ is an independent Nakagami fading variable of the $i$-th interferer and $N$ is the number of co-channel interferers in the system. The PDF of the SIR of our system, i.e., $f_{\gamma}(r)$, is determined by using the formula

$$
\begin{aligned}
& f_{\gamma}(r)=\int_{0}^{\infty} x f_{S}(r x) f_{I}(x) d x[37] \text { as } \\
& f_{\gamma}(r)=\int_{0}^{\infty} x \underbrace{\frac{(r x)^{\frac{\delta}{2}-1} e^{-\frac{\sqrt{r x}}{\rho}}}{2 \rho^{\delta} \Gamma(\delta)}}_{f_{S}(r x)} \times \underbrace{\frac{\omega^{m_{T}} x^{m_{T}-1}}{\Gamma\left(m_{T}\right)} \exp (-\omega x)}_{f_{I}(x)} d x, \\
& f_{\gamma}(r)= \frac{2^{-\left(\delta+2 m_{T}\right)} \omega^{-\frac{\delta}{2}} \Gamma\left(\delta+2 m_{T}\right)}{\rho^{\delta} \Gamma(\delta) \Gamma\left(m_{T}\right)} r^{\frac{\delta}{2}-1} \\
& \times \psi\left(\frac{\delta+2 m_{T}}{2}, \frac{1}{2}, \frac{r}{4 \omega \rho^{2}}\right)
\end{aligned}
$$

In (4), $\delta$ and $\rho$ are the shape and the scale parameters of the desired signal, respectively, $\psi($.$) is KummerU function [33]$ and $m_{T}=N m$, where $m$ is the fading parameter for the interferer signals. Also, $\omega=\beta / g$, where $\beta=m / \Omega$ in which $\Omega$ is the average power of the interference fading channel. Based on (4), the expression of cumulative distribution function (CDF) of our system, after some mathematical manipulation, is given as

$$
\begin{aligned}
& F_{\gamma}(r)=\frac{2^{-\left(\delta+2 m_{T}\right)} \omega^{-\frac{\delta}{2}} \Gamma\left(\delta+2 m_{T}\right) r^{\frac{\delta}{2}}}{\rho^{\delta} \Gamma(\delta) \Gamma\left(m_{T}\right) \Gamma\left(\frac{\delta+2 m_{T}}{2}\right) \Gamma\left(\frac{\delta+2 m_{T}}{2}+\frac{1}{2}\right)}
\end{aligned}
$$

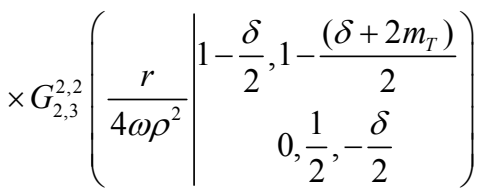

In (5), $G_{y, z}^{w, x}$ is the Meijer-G function [33]. The probability of drop-down of the SIR of a system below a predefined threshold $\mathrm{R}$ is termed as the outage probability. By using $P_{\text {out }}=\int_{0}^{R} f_{\gamma}(r) d r[38]$ the expression for the outage probability of our D2D communication system is :

$$
\begin{aligned}
P_{\text {out }}= & \frac{2^{-\left(\delta+2 m_{T}\right)} \omega^{-\frac{\delta}{2}} \Gamma\left(\delta+2 m_{T}\right) R^{\frac{\delta}{2}}}{\rho^{\delta} \Gamma(\delta) \Gamma\left(m_{T}\right) \Gamma\left(\frac{\delta+2 m_{T}}{2}\right) \Gamma\left(\frac{\delta+2 m_{T}}{2}+\frac{1}{2}\right)} \\
& \times G_{2,3}^{2,2}\left(\frac{R}{4 \omega \rho^{2}} \mid \begin{array}{c}
1-\frac{\delta}{2}, 1-\frac{\left(\delta+2 m_{T}\right)}{2} \\
0, \frac{1}{2},-\frac{\delta}{2}
\end{array}\right)
\end{aligned}
$$

By using the channel capacity expression $C=\int_{0}^{\infty} \log _{2}(1+r) f_{\gamma}(r) d r[41]$, the expression for the channel capacity of our D2D communication system is:

$$
\begin{aligned}
C= & \frac{2^{-\left(\delta+2 m_{T}\right)} \omega^{-\frac{\delta}{2}} \Gamma\left(\delta+2 m_{T}\right)}{\ln (2) \rho^{\delta} \Gamma(\delta) \Gamma\left(m_{T}\right) \Gamma\left(\frac{\delta+2 m_{T}}{2}\right) \Gamma\left(\frac{\delta+2 m_{T}}{2}+\frac{1}{2}\right)} \\
& \times G_{3,4}^{4,2}\left(\frac{1}{4 \omega \rho^{2}} \mid \begin{array}{c}
1-\frac{\left(\delta+2 m_{T}\right)}{2},-\frac{\delta}{2}, 1-\frac{\delta}{2} \\
0, \frac{1}{2},-\frac{\delta}{2},-\frac{\delta}{2}
\end{array}\right)
\end{aligned}
$$

The SER performance expression for the D2D system incorporating $M$-ary phase-shift keying ( $M$-PSK) modulation, after some mathematical manipulations, is [33]: 


$$
\begin{aligned}
P_{e} & =\frac{2^{-\left(\delta+2 m_{T}\right)} \omega^{-\frac{\delta}{2}} \Gamma\left(\delta+2 m_{T}\right) \Gamma\left(\frac{\delta}{2}\right) \Gamma\left(\frac{\delta+1}{2}\right)}{\pi \rho^{\delta} \Gamma(\delta) \Gamma\left(m_{T}\right) \Gamma\left(\delta+m_{T}+\frac{1}{2}\right)\left(\sin \frac{\pi}{M}\right)^{\delta}} \\
& \times \int_{0}^{\frac{(M-1) \pi}{M}}(\sin \theta)^{\delta}{ }_{2} F_{1}\left(\frac{\delta}{2}, \frac{\delta+2 m_{T}}{2} ; \delta+m_{T}+\frac{1}{2} ; 1-\frac{\left(\frac{\sin \theta}{\sin \frac{\pi}{M}}\right)^{2}}{4 \omega \rho^{2}}\right)
\end{aligned}
$$

where $M$ is the order of modulation and ${ }_{2} F_{1}$ is hypergeometric function [33].

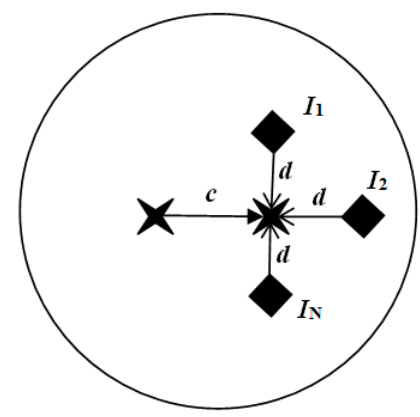

Fig. 1. D2D communication system layout

where

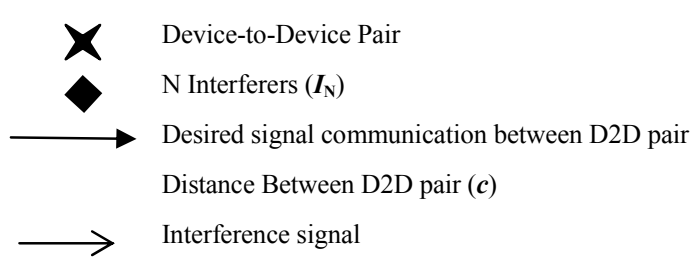

Distance Between an Interferer and D2D Receiver $(\boldsymbol{d})$

\section{NUMERICAL RESULTS}

In this section, numerical results are presented and discussed based on the expressions in Section II. Our expressions are valid for arbitrary values of the channel and interference parameters. For the analysis the reference distances $c_{0}$ and $d_{0}$ are assumed to be $1 \mathrm{~m}$. For the outage probability analysis, the SIR threshold is assumed to be $10 \mathrm{dBm}$. The outage performance of our D2D communication system is shown in Figure 2. The values of $P_{1}, \delta$ and $a$, are assumed to be $20 \mathrm{dBm}, 3$ and 3 , respectively. The values of $P_{2}$, $b$ and $m$ are considered to be $13 \mathrm{dBm}, 2.8$ and 2, respectively. We consider 5 interferers in the system. From the figure, we observe that as the distance between D2D devices increases the outage performance degrades due to worsening of SIR performance. We also observe that as interferers move away from the receiver the outage performance improves.

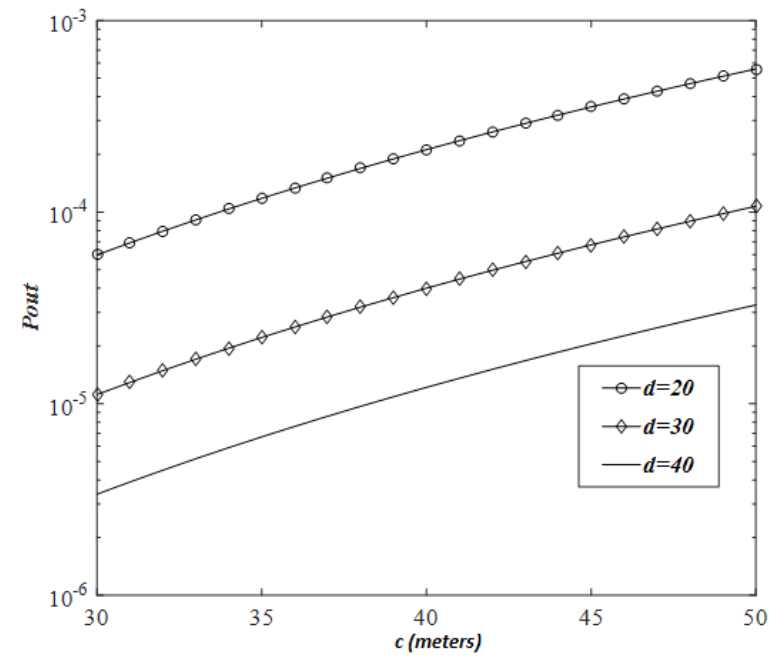

Fig. 2. Outage probability comparison with varying distance between the receiver and the interferers

Figure 3 shows the outage performance with varying fading conditions for the interference. The values of $P_{1}, \delta, P_{2}, a, b$ and $d$ are considered to be $20 \mathrm{dBm}, 3,13 \mathrm{dBm}, 3.5,2.7$ and $50 \mathrm{~m}$, respectively. We consider 5 interferers in the system. From the figure, we observe negligible change in the outage performance of our system when the interference channel fading conditions are varied. Hence, our system is mostly insensitive to the variations in the fading conditions of the interferers. Figure 4 shows the outage performance with varying path-loss exponent values of the interfering signals, $b$. The values for $P_{1}, \delta, P_{2}, m$, $a$ and $d$ are fixed at $20 \mathrm{dBm}, 3,13 \mathrm{dBm}, 2,3.5$ and $50 \mathrm{~m}$, respectively. We consider 5 interferers in the system. From the figure, it can be observed that by increasing the path-loss exponent value of the interference signal, the outage performance improves. It is due to the weakening effect of the interference signal strength at the receiver when path-loss exponent value is increased. There is an inverse relation between the received signal strength and the path-loss exponent value. Channel capacity performance of our system with varying numbers of co-channel interferers and D2D signal power is shown in Figure 5. The values for $\delta, a, m, b, c, d$ and $P_{2}$ are considered to be $3,2.7,2,3,60 \mathrm{~ms}, 30 \mathrm{~m}$ and $16 \mathrm{dBm}$, respectively. From the Figure, it can be seen that when the strength of the D2D signal is increased, capacity performance improves due to the improved SIR conditions of the system. However, when the number of interferers in the system is increased, capacity performance deteriorates due to degraded SIR conditions. Channel capacity performance comparison with varying path-loss exponent values of co-channel interferers is shown in Figure 6. We also vary the number of interferers in the system. The values for $P_{1}, \delta, a, m, b, c, d$ and $P_{2}$ are considered to be $20 \mathrm{dBm}, 3,2.8,2,3.5,60 \mathrm{~m}, 30 \mathrm{~m}$ and $16 \mathrm{dBm}$, respectively. It can be observed that by increasing the path-loss exponent values of co-channel interferers the capacity performance improves. It is due to the weakening of the interference signals when path-loss exponent value increases. 


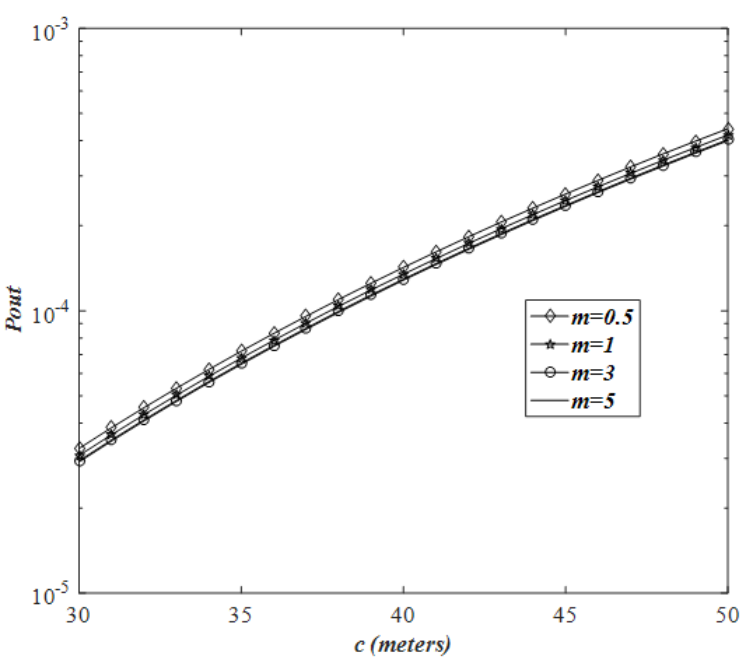

Fig. 3. Outage performance comparison with varying fading parameters of the interferers.

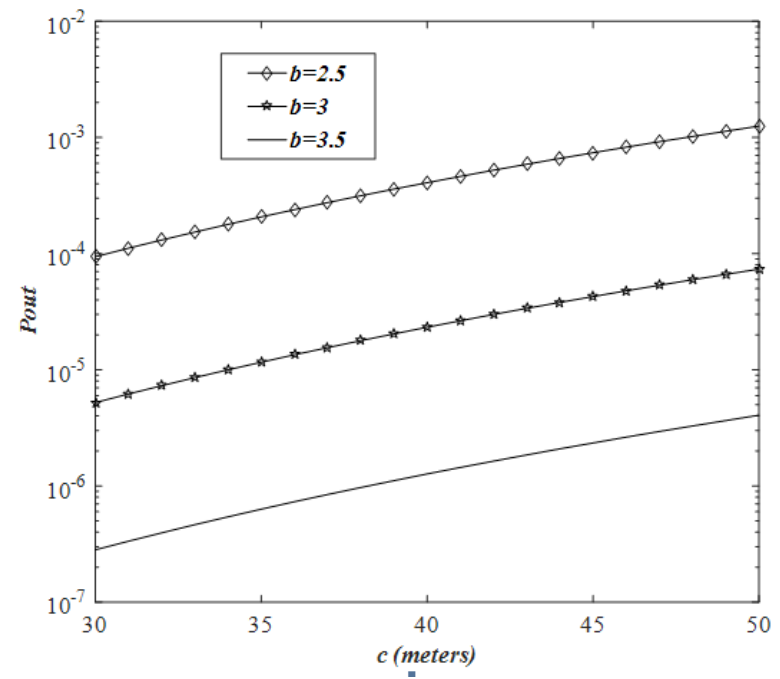

Fig. 4. Outage probability comparison with varying path-loss exponent values of the interferers.

Performance analysis of 8-PSK modulated D2D system with varying values of shape parameter and path-loss exponents of desired signal is shown in Figure 7. The values of $P_{1}, P_{2}, m, b, c$ and $d$ are assumed to be $20 \mathrm{dBm}, 13 \mathrm{dBm}, 2,4,30$ $\mathrm{m}$ and $70 \mathrm{~m}$, respectively. We consider 5 interferers in the system. From the figure, we observe that the SER performance degrades as the path-loss exponent value is increased. |This is due to the weakening of the received D2D signal. We also observe that under better channel fading conditions SER performance of our system is improved. Performance analysis of 8-PSK system with varying D2D signal power and D2D signal path-loss exponent values is shown in Figure. 8. The values for $P_{2}, m, b, c$ and $d$ are assumed to be $13 \mathrm{dBm}, 2,3.5$, $30 \mathrm{~m}$ and $70 \mathrm{~m}$, respectively. We consider 5 interferers in the system. It can be seen from the figure that when the D2D signal strength is increased, improved SER performance is observed due to better SIR conditions.

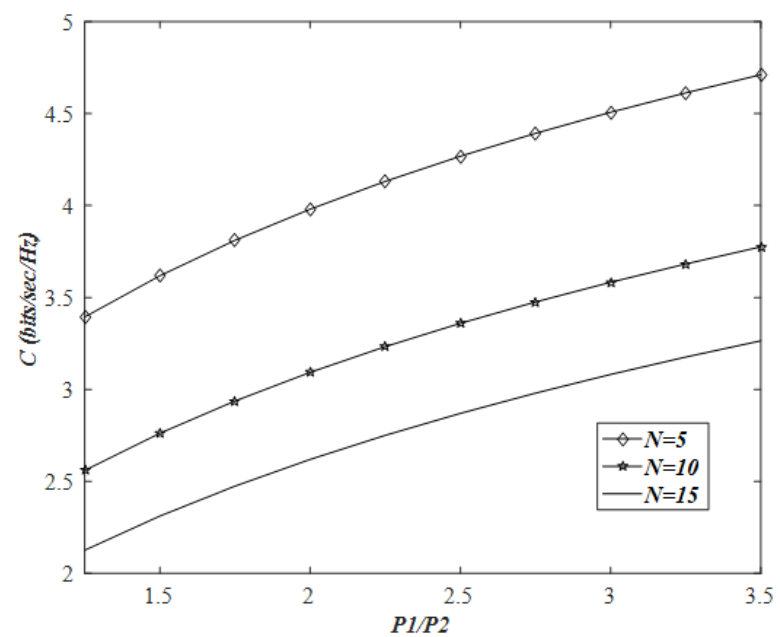

Fig. 5. Capacity performance comparison with varying number of cochannel interferers

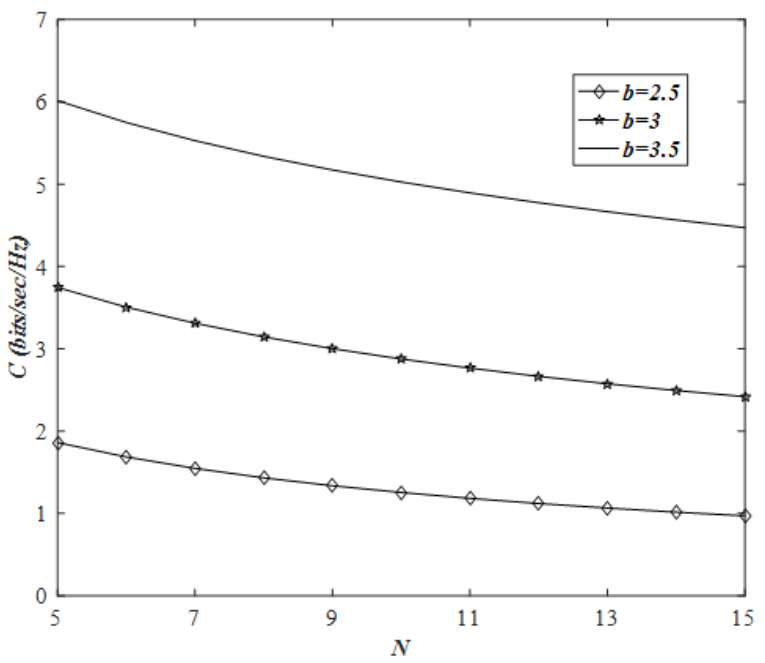

Fig. 6. Capacity performance with varying number of path-loss exponent values of interferers

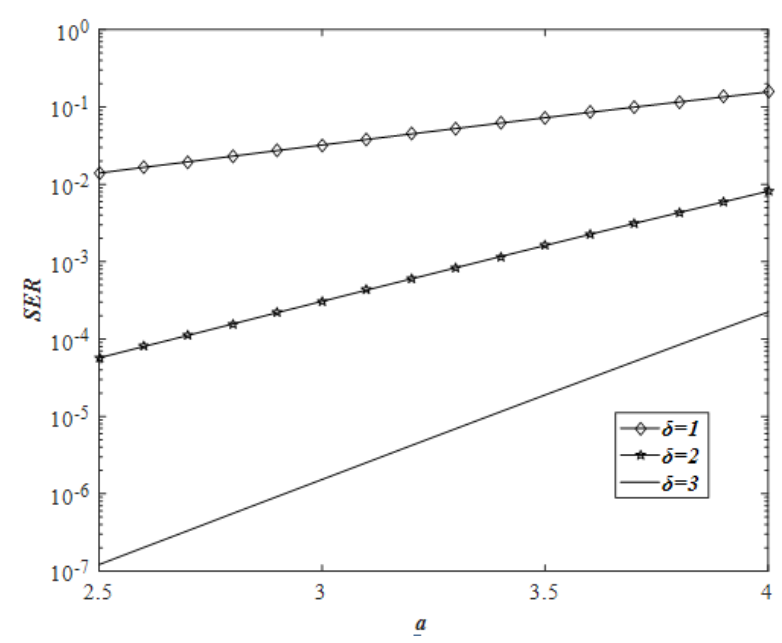

Fig. 7. SER performance of 8-PSK system with varying shape parameters of D2D signal 


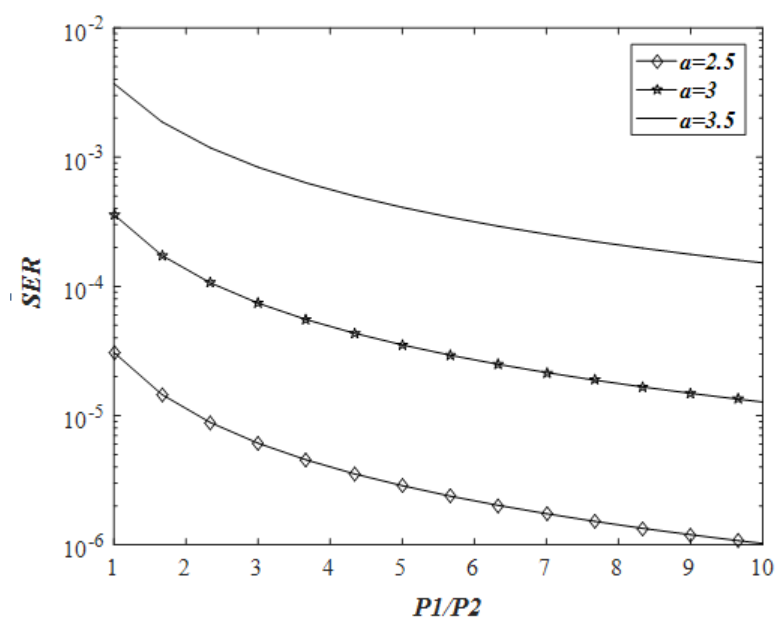

Fig. 8. SER performance of 8-PSK system with varying path-loss exponents of D2D signal

\section{CONCLUSION}

We studied and analyzed the outage probability, channel capacity and SER performances of D2D communication systems over gamma fading channels in the presence of multiple Nakagami faded co-channel interferers. We also considered path-loss effects in our system. We presented a PDF of the SIR of our system. Based on this PDF, expressions for the outage probability, channel capacity and SER are presented. With the help of numerical results based on our expressions, we discussed the effects of various channel parameters, like fading and path-loss, on the outage, channel capacity and SER performances of our D2D communication system. We also observed that our D2D communication system performance is largely insensitive to the variations in the channel fading conditions of the interferers. However, variation in the pathloss exponent values of the interference signal show variation in the overall D2D system performance.

\section{REFERENCES}

[1] C. Gao, H. Zhang, X. Chen, Y. Li, D. Jin, S. Chen, "Impact of Selfishness in Device-to-Device Communication Underlaying Cellular Networks", IEEE Transactions on Vehicular Technology, Vol. 66, No.10, pp. 9338-0349, 2017

[2] Z. Zhou, C. Gao, C. Xu, T. Chen, D. Zhang, S. Mumtaz, "EnergyEfficient Stable Matching for Resource Allocation in Energy Harvesting-Based Device-to-Device Communications", IEEE Access, Vol. 5, pp. 15184-15196, 2017

[3] P. Zhao, L. Feng, P. Yu, W. Li, X. Qiu, "A Social-Aware Resource Allocation for 5G Device-to-Device Multicast Communication", IEEE Access, Vol. 5, pp. 15717-15730, 2017

[4] A. Ramezani-Kebrya, M. Dong, B. Liang, G. Boudreau, S. H. Seyedmehdi, "Joint Power Optimization for Device-to-Device Communication in Cellular Networks with Interference Control", IEEE Transactions on Wireless Communications, Vol. 16, No. 8, pp. 51315146, 2017

[5] Y. J. Chun, S. L. Cotton, H. S. Dhillon, A. Ghrayeb, M. O. Hasna, "A stochastic geometric analysis of device-to-device communications operating over generalized fading channels", IEEE Transactions on Wireless Communications, Vol. 16, No. 7, pp. 4151-4165, 2017

[6] S. M. A. Kazmi, N. H. Tran, W. Saad, Z. Han, T. M. Ho, T. Z. Oo ,C. S. Hong, "Mode Selection and Resource Allocation in Device-to-Device
Communications: A Matching Game Approach", IEEE Transactions on Mobile Computing, Vol. 16, No.11, pp. 3126-3141, 2017

[7] J. Park, J. H. Lee, "Semi-Distributed Spectrum Access to Enhance Throughput for Underlay Device-to-Device Communications", IEEE Transactions on Communications, Vol. 65, No. 10, pp.4572-4582, 2017

[8] L. Fan, Z. Dong, P. Yuan, The Capacity of Device-to-Device Communication Underlaying Cellular Networks With Relay Links, IEEE Access, Vol. 5, pp.16840-16846, 2017

[9] Y. Xiao, D. Niyato, K. C. Chen, Z. Han, "Enhance device-to-device communication with social awareness: A belief-based stable marriage game framework", IEEE Wireless Communications, Vol. 23, No. 4, pp.36-44, 2016

[10] Y. Li, D. Jin, P. Hui, Z. Han, "Optimal base station scheduling for device-to-device communication underlaying cellular networks", IEEE Journal on Selected Areas in Communications, Vol. 34, No. 1, pp.27-40, 2016

[11] C. Y. Wang, G. Y. Lin, C. C. Chou, C. W. Yeh, H. Y. Wei, "Device-toDevice Communication in LTE-Advanced System: A Strategy-proof Resource Exchange Framework", IEEE Transactions on Vehicular Technology, Vol. 65, No. 12, pp.10022-10036, 2016

[12] B. Zhong, J. Zhang, Q. Zeng, X. Dai, "Coverage probability analysis for Full-Duplex relay aided Device-to-Device communications networks", China Communications Vol. 13, No. 11, pp.60-67, 2016

[13] H. Meshgi, D. Zhao and R. Zheng, "Optimal Resource Allocation in Multicast Device-to-Device Communications Underlaying LTE Networks", IEEE Transactions on Vehicular Technology, Vol. 66, No. 9, pp. 8357-8371, 2017

[14] J. Liu, N. Kato, J. Ma, N. Kadowaki, "Device-to-device communication in LTE-advanced networks: A survey", IEEE Communications Surveys \& Tutorials, Vol. 17, No. 4, pp.1923-1940, 2015

[15] T. Huynh, T. Onuma, K. Kuroda, M. Hasegawa, W. J. Hwang, "Joint downlink and uplink interference management for device to device communication underlaying cellular networks", IEEE Access, Vol. 4, pp. 4420-44230, 2016

[16] N. Rajkumar, M. Lakshmanan, V. N. Mohammed, M. Palanivelan, "Distance based uplink resource sharing for device-to-device communication", 4th International Conference on Electronics and Communication Systems, Coimbatore, India, pp. 74-77, February 24-25, 2017

[17] A. Asadi, Q. Wang, V. Mancuso, "A survey on device-to-device communication in cellular networks", IEEE Communications Surveys \& Tutorials, Vol. 16, No. 4, pp. 1801-1819, 2014

[18] H. W. Tseng, Y. J. Yu, B. S. Wu,C. F. Kuo, P. S. Chen, “A resource allocation scheme for device-to-device communication over ultra-dense 5G cellular networks", International Conference on Applied System Innovation, Sapporo, Japan, pp. 80-83, May 13-17, 2017

[19] X. Lin, J. Andrews, A. Ghosh, R. Ratasuk, "An overview of 3GPP device-to-device proximity services”, IEEE Communications Magazine, Vol. 52, No. 4, pp. 40-48, 2014

[20] H. Ghavami,S. S. Moghaddam, "Outage probability for underlaying Device to Device communications", 8th International Symposium on Telecommunications, Tehran, Iran, pp. 353-358, September 27-28, 2016

[21] S. Xu, H. Zhang, J. Tian, S. Guo, X. Zhou, "Distributed energy-efficient resource allocation and power control for device-to-device communications underlaying cellular networks", 19th International Symposium on Wireless Personal Multimedia Communications, Shenzhen, China, pp. 441-446, November 14-16, 2016

[22] K. Yang, J. Wu, X. Gao, X. Bu, S. Guo, "Energy-Efficient Power Control for Device-to-Device Communications with Max-Min Fairness", 84th IEEE Vehicular Technology Conference, Montreal, Canada, pp. 1-5, September 18-21, 2016

[23] K. Yang, S. Martin, C. Xing, J. Wu, R. Fan, "Energy-Efficient Power Control for Device-to-Device Communications", IEEE Journal on Selected Areas in Communications,Vol. 34, No.12, pp. 3208-3220, 2016

[24] C. P. Liu , C. G. He, W. X. Meng, "A Tractable Multi-RATs Offloading Scheme on D2D Communications", IEEE Access, Vol. 5, pp. 2084120851,2017 
[25] N. Senadhira, J. Guo, S. Durrani, "Outage analysis of underlaid multiantenna D2D communication in cellular networks", 10th International Conference on Signal Processing and Communication Systems, Gold Coast, Australia, pp. 1-7, December 19-21, 2016

[26] J. Liu, H. Nishiyama, N. Kato, J. Guo, "On the outage probability of device-to-device-communication-enabled multichannel cellular networks: An RSS-threshold-based perspective", IEEE Journal on Selected Areas in Communication, Vol. 34, No. 1, pp. 163-175, 2016

[27] H. Ghavami, S. S. Moghaddam, "Outage Probability of Device to Device Communications Underlaying Cellular Network in Suzuki Fading Channel", IEEE Communications Letters,Vol. 21, No. 5, pp.1203-1206, 2017

[28] C. Liu, B. Natarajan, "Ergodic capacity in D2D underlay networks with varying user distribution", 13th IEEE Annual Consumer Communications \& Networking Conference, Las Vegas, USA, pp. 325329, January 9-12, 2016

[29] M. Lin, J. Ouyang, W. P. Zhu, "Joint beamforming and power control for device-to-device communications underlaying cellular networks", IEEE Journal on Selected Areas in Communications, Vol. 34, No. 1, pp. $138-150,2016$

[30] A. Chopra, B. L. Evans, "Outage probability for diversity combining in interference-limited channels", IEEE Transactions on Wireless Communications, Vol. 12, No. 2, pp.550-560, 2013

[31] S. S. Nam, Y. Choi, M. S. Alouini, S. Choi, "Impact of Self-Interference on the Performance of Joint Partial RAKE Receiver and Adaptive Modulation", IEEE Access, Vol. 4, pp. 8525-8534, 2016

[32] H. Popovic, D. Stefanovic, A. Mitic, I. Stefanovic, D. Stefanovic, "Some statistical characteristics of Nakagami-m distribution", 8th International Conference on Telecommunications in Modern Satellite, Cable and Broadcasting Services, Nis, Serbia, pp. 509-512, September 26-28, 2007

[33] I. S. Gradshteyn, I. M. Ryzhik, Table of Integrals, Series, and Products, Academic Press, 2007

[34] M. Elalem, L. Zhao, "Effective capacity optimization for cognitive radio network based on underlay scheme in gamma fading channels", International Conference on Computing, Networking and Communications, San Diego, USA, pp. 714-718, January 28-31, 2013

[35] A. Maaref, R. Annavajjala, "The Gamma Variate with Random Shape Parameter and Some Applications", IEEE Communication Letters, Vol. 14, No. 12, pp. 1146-1148, 2010

[36] A. Goldsmith, Wireless Communications, Cambridge University Press, 2005

[37] I. Trigui, A. Laourine, S. Affes,A. Stephenne, "Outage Analysis of Wireless Systems over Composite Fading/Shadowing Channels with CoChannel Interference", IEEE Wireless Communications and Networking Conference, Budapest, Hungary, pp. 1-6, April 5-8, 2009

[38] R. M. Radaydeh, "SNR and SINR-based selection combining algorithms in the presence of arbitrarily distributed co-channel interferers", IET Communications, Vol. 3, No. 1, pp. 57-66, 2009

[39] M.-S. Alouini, A. J. Goldsmith, "Capacity of Rayleigh fading channels under different adaptive transmission and diversity-combining techniques", IEEE Transactions on Vehicular Technology, Vol. 48, No. 4, pp. 1165-1181, 1999 